Acta Crystallographica Section E

Structure Reports

Online

ISSN 1600-5368

\section{8,10-Diiodo-2,6-dioxo-4 $\lambda^{3}$-ioda-3,5- dioxatricyclo[5.3.1.0 $\left.{ }^{4,11}\right]$ undeca- 1(11),7,9-triene-9-carboxylic acid}

\section{Daopeng Sheng, Lu Han, Yi Zhang and Yanzhao Yang*}

Key Laboratory for Special Functional Aggregated Materials of the Education Ministry, School of Chemistry and Chemical Engineering, Shandong University, Jinan 250100, People's Republic of China

Correspondence e-mail: yzhyang@sdu.edu.cn

Received 5 January 2012; accepted 7 February 2012

Key indicators: single-crystal X-ray study; $T=130 \mathrm{~K}$; mean $\sigma(\mathrm{C}-\mathrm{C})=0.010 \AA$; $R$ factor $=0.042 ; w R$ factor $=0.097 ;$ data-to-parameter ratio $=16.6$.

In the title compound, $\mathrm{C}_{9} \mathrm{HI}_{3} \mathrm{O}_{6} \cdot 2 \mathrm{H}_{2} \mathrm{O}$, the molecule is located on a twofold axis that gives rise to disorder of the carboxyl group. This disorder is correlated with the disorder of one of the $\mathrm{H}$ atoms of the water molecule. The carboxyl group is twisted relative to the attached benzene ring by $75.1(4)^{\circ}$. The intramolecular I...O distance is 2.112 (6) §. Molecules are linked via $\mathrm{O}-\mathrm{H} \cdots \mathrm{O}$ hydrogen bonding, $\mathrm{C}-\mathrm{I} \cdots \mathrm{O}$ halogen bonding, with $\mathrm{I} \cdots \mathrm{O}$ distances in the range $3.156(5)-$ 3.274 (6) $\AA$, and dipolar $\mathrm{C}=\mathrm{O} \cdots \mathrm{C}=\mathrm{O}$ interactions between the carboxyl and carboxylate groups, with an $\mathrm{O} \cdots \mathrm{C}$ distance of 2.944 (10) $\AA$.

\section{Related literature}

For general background to 1,3,5-triiodobenzene derivatives, see: Morin et al. (1987); Yu \& Watson (1999). For information on the related compound 1,3,5-triiodo-2,4,6-trimethylbenzene, see: Bosch \& Barnes (2002); Boudjada et al. (2001); Reddy et al. (2006). For the crystal structures of 5-amino-2,4,6-triiodoisophthalic acid monohydrate and 5-amino-2,4,6-triiodoisophthalic acid-4,4'-bipyridine $N, N^{\prime}$-dioxide-water (1/1/1), see: Beck \& Sheldrick (2008); Zhang et al. (2011).

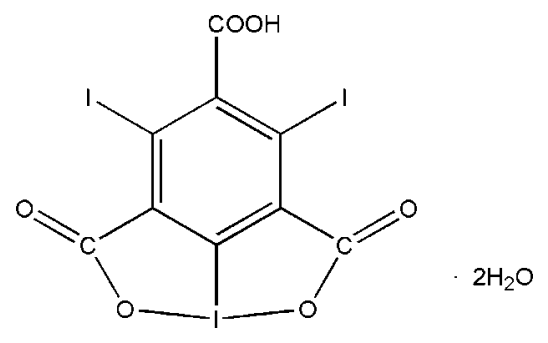

\section{Experimental}

Crystal data

$\mathrm{C}_{9} \mathrm{HI}_{3} \mathrm{O}_{6} \cdot 2 \mathrm{H}_{2} \mathrm{O}$

$M_{r}=621.83$

Monoclinic, $C 2 / c$

$a=14.7667$ (8) ̊

$b=11.9890(6) \AA$

$c=9.7419(5) \AA$

$\beta=127.4236(5)^{\circ}$

$V=1369.68(12) \AA^{3}$

$Z=4$

Mo $K \alpha$ radiation

$\mu=6.88 \mathrm{~mm}^{-1}$

$T=130 \mathrm{~K}$

$0.32 \times 0.14 \times 0.12 \mathrm{~mm}$

\section{Data collection}

Bruker APEXII CCD area-detector diffractometer

Absorption correction: multi-scan (APEX2; Bruker, 2007)

$T_{\min }=0.217, T_{\max }=0.492$

Refinement

$R\left[F^{2}>2 \sigma\left(F^{2}\right)\right]=0.042$

$w R\left(F^{2}\right)=0.097$

$S=1.23$

1547 reflections

$\mathrm{H}$-atom parameters constrained

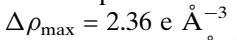

$\Delta \rho_{\min }=-2.23 \mathrm{e}^{-3}$

Table 1

Hydrogen-bond geometry $\left(\AA,{ }^{\circ}\right)$.

\begin{tabular}{|c|c|c|c|c|}
\hline$D-\mathrm{H} \cdots A$ & $D-\mathrm{H}$ & $\mathrm{H} \cdots A$ & $D \cdots A$ & $D-\mathrm{H} \cdots A$ \\
\hline $\mathrm{O} 3-\mathrm{H} 3 \cdots \mathrm{O} 1 W$ & 0.82 & 2.08 & $2.772(9)$ & 142 \\
\hline $\mathrm{O} 1 W-\mathrm{H} 1 W \cdots \mathrm{O} 3$ & 0.82 & 1.98 & $2.772(9)$ & 163 \\
\hline $\mathrm{O} 1 W-\mathrm{H} 2 W \cdots \mathrm{O} 1 W^{\mathrm{i}}$ & 0.82 & 1.94 & $2.730(14)$ & 160 \\
\hline $\mathrm{O} 1 W-\mathrm{H} 3 W \cdots \mathrm{O} 1^{\mathrm{ii}}$ & 0.82 & 2.24 & $3.053(9)$ & 172 \\
\hline
\end{tabular}

Symmetry codes: (i) $-x+1,-y+2,-z+1$; (ii) $-x+\frac{1}{2}, y+\frac{1}{2},-z+\frac{1}{2}$.

Data collection: APEX2 (Bruker, 2007); cell refinement: SAINT (Bruker, 2007); data reduction: $S A I N T$; $\operatorname{program}(\mathrm{s})$ used to solve structure: SIR97 (Altomare et al., 1999); program(s) used to refine structure: SHELXL97 (Sheldrick, 2008); molecular graphics: DIAMOND (Brandenburg, 1999); software used to prepare material for publication: publCIF (Westrip, 2010).

The authors acknowledge financial support for this work from the National Natural Science Foundation of China (grant Nos. 20876089 and 21076115), the Natural Science Foundation of Shandong Province (grant No. ZR2010BM019) and the 973 Project of China (grant No. 2011CB935901).

Supplementary data and figures for this paper are available from the IUCr electronic archives (Reference: GK2447).

\section{References}

Altomare, A., Burla, M. C., Camalli, M., Cascarano, G. L., Giacovazzo, C., Guagliardi, A., Moliterni, A. G. G., Polidori, G. \& Spagna, R. (1999). J. Appl. Cryst. 32, 115-119.

Beck, T. \& Sheldrick, G. M. (2008). Acta Cryst. E64, o1286.

Bosch, E. \& Barnes, C. L. (2002). Cryst. Growth Des. 2, 299-302.

Boudjada, A., Hernandez, O., Meinnel, J., Mani, M. \& Paulus, W. (2001). Acta Cryst. C57, 1106-1108.

Brandenburg, K. (1999). DIAMOND. Crystal Impact GbR, Bonn, Germany. Bruker (2007). APEX2 and SAINT. Bruker AXS Inc., Madison, Wisconsin, USA.

Morin, J. P., Boutelet, I., Toutain, H. \& Fillastre, J. P. (1987). Pathol. Biol. 35, 1215-1220.

Reddy, C. M., Kirchner, M. T., Gundakaram, R. C., Padmanabhan, K. A. \& Desiraju, G. R. (2006). Chem. Eur. J. 12, 2222-2234.

Sheldrick, G. M. (2008). Acta Cryst. A64, 112-122.

Westrip, S. P. (2010). J. Appl. Cryst. 43, 920-925.

Yu, S. B. \& Watson, A. D. (1999). Chem. Rev. 99, 2353-2378.

Zhang, K.-L., Zhang, J.-B. \& Ng, S. W. (2011). Acta Cryst. E67, o793. 


\section{supporting information}

Acta Cryst. (2012). E68, o706 [doi:10.1107/S1600536812005351]

\section{8,10-Diiodo-2,6-dioxo-4 $\lambda^{3}$-ioda-3,5-dioxatricyclo[5.3.1.0 $\left.{ }^{4,11}\right]$ undeca-1(11),7,9- triene-9-carboxylic acid}

\section{Daopeng Sheng, Lu Han, Yi Zhang and Yanzhao Yang}

\section{S1. Comment}

Iodine-based compounds have always been used as contrast agents for X-ray imaging (Morin et al., 1987). The 1,3,5-triiodo-benzene core has been the basis of many contrast agents (Yu \& Watson, 1999). In this paper, we present the crystal structure of a new compound based on 1,3,5-triiodobenzene core.

In the title compound the organic molecule is located on a twofold axis what results in disorder of the carboxylic group (Fig. 1). In the crystal structure, there are hydrogen bonds between symmetry related water molecules as well as between the water molecule and the carboxylic group. It indicates that one of the hydrogen atoms of the water molecule has to be disordered and this disorder is evidently correlated with the disorder of the carboxylic group. The hydrogen atom and the oxygen atom forming hydrogen bond between the water molecule and the carboxylic group are either from the water molecule or the carboxylic group. There is also a hydrogen bond between the water molecule and the carboxylate $\mathrm{O} 1$ atom. Hydrogen atom involved in this interaction has full occupancy. The dihedral angle between the plane of the carboxyl group and the benzene ring is $75.1(4)^{\circ}$.

In addition to hydrogen bond, the structure is stabilized by halogen bonding between the I 2 atom and the carboxylate group $\mathrm{O} 1$ and $\mathrm{O} 2$ atoms. There is also a halogen bond between the water molecule and I1 atom (Fig. 2). A dipolar interaction between carboxyl $\mathrm{C} 6-\mathrm{O} 3$ and carboxylate $\mathrm{C} 4$ also is observed (C‥O $2.95 \AA$ ) (Fig. 3).

\section{S2. Experimental}

A mixture of 1,3,5-triiodo-2,4,6-trimethylbenzene ( $5 \mathrm{~g})$ and excess of potassium permanganate $(80 \mathrm{~g})$ was dissolved in pyridine $(60 \mathrm{ml})$ and heated under reflux for $24 \mathrm{~h}$ to produce the title compound (m.p. $573 \mathrm{~K}$, decompose). Crystallization was carried out from a mixture of water and methanol ( $v / v 1: 2)$. Colorless crystals suitable for X-ray single-crystal diffraction were obtained by slow evaporation method.

\section{S3. Refinement}

$\mathrm{H}$ atom of the carboxylic group was placed in geometrically calculated position and refined using a riding model the the occupantion factor of 0.5. Positions of $\mathrm{H}$ atoms from the water molecule were calculated after analysis of possible hydrogen-bond interactions. The occupantion factors of $\mathrm{H} 1 \mathrm{~W}$ and $\mathrm{H} 2 \mathrm{~W}$ were assigned as 0.5 .

The isotropic displacement parameters of all $\mathrm{H}$ atoms were set to 1.5 times the equivalent displacement parameter of their parent $\mathrm{O}$ atoms. 


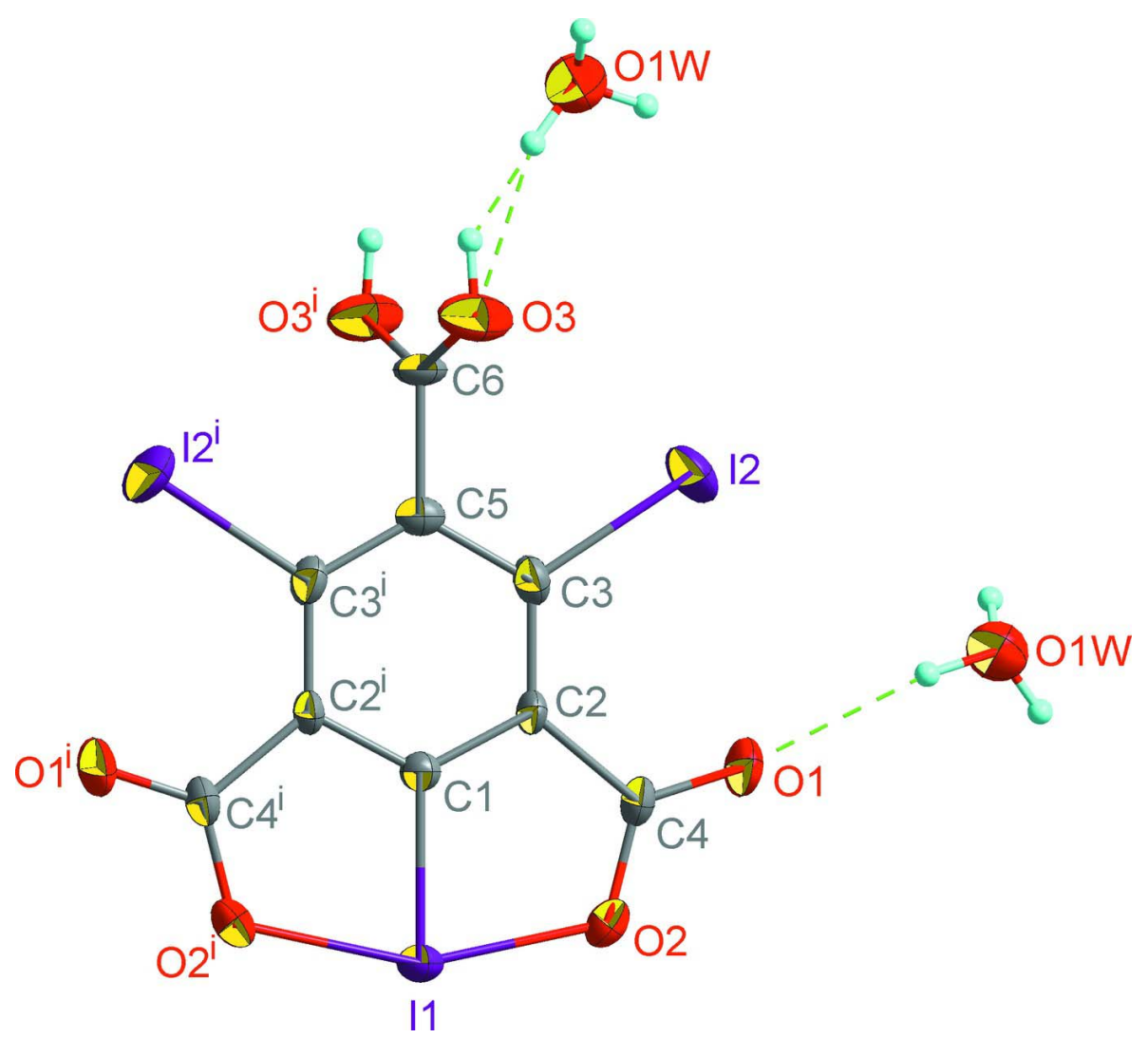

\section{Figure 1}

Molecular structure of the title compound. Displacement ellipsoids are drawn at the 50\% probability level. Symmetry code: (i) $1-x, y, 1.5+z$.

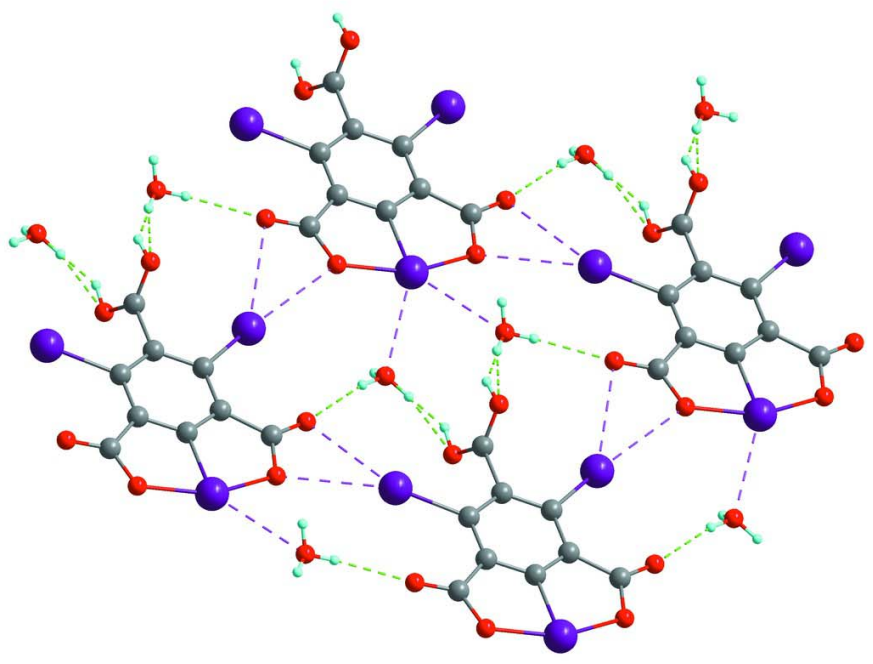

Figure 2

Partial view of the crystal structure. Molecules are linked by $\mathrm{O}-\mathrm{H} \cdots \mathrm{O}$ (green dashed lines) and $\mathrm{O}-\mathrm{H} \cdots \mathrm{I}$ hydrogen bonds (purple dashed lines). 


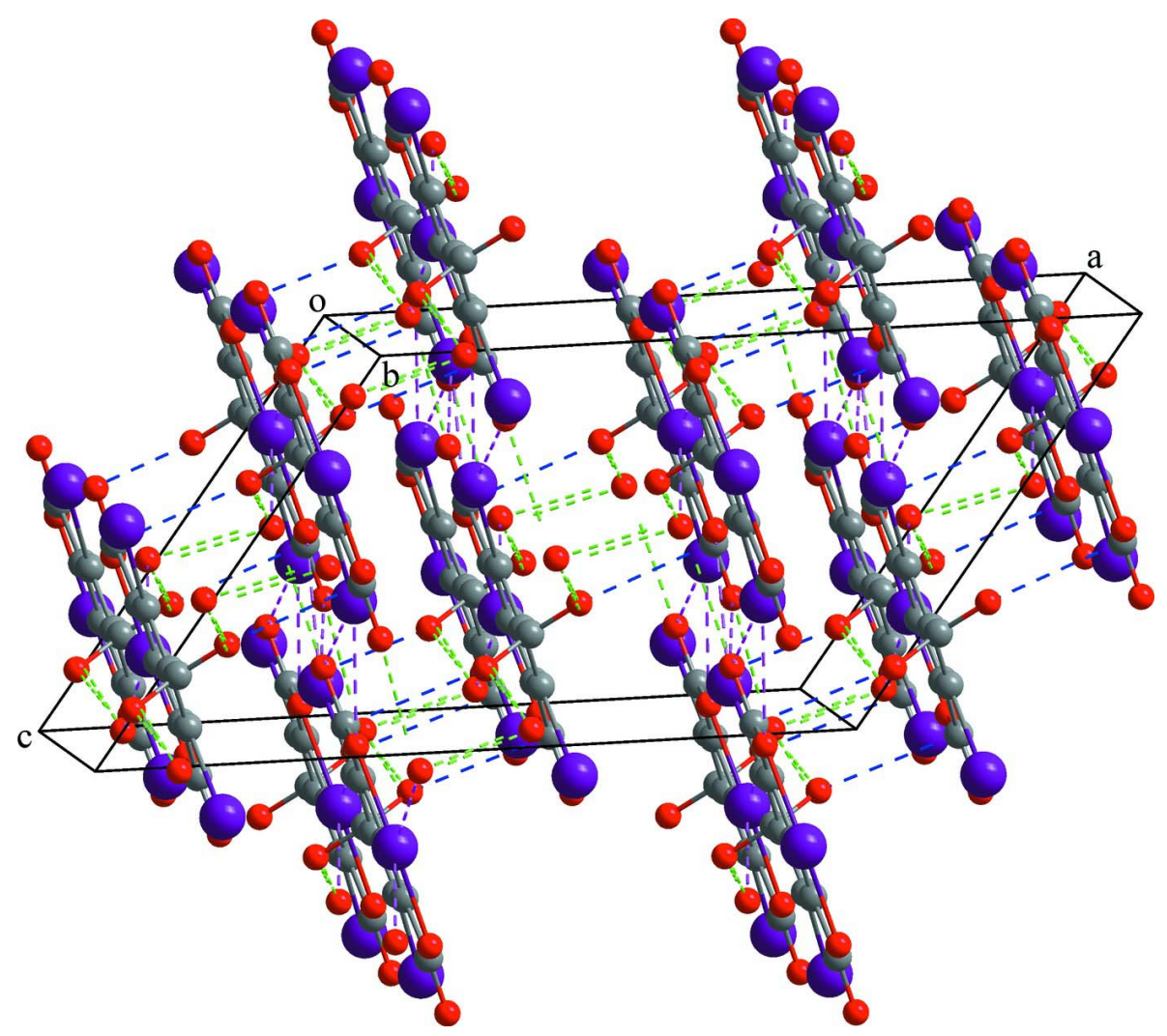

Figure 3

Packing of the title compound, hydrogen atoms are omitted for clarity. Hydrogen bonds (green), halogen bonds (purple) and dipolar interaction (blue) are shown as dashed lines.

\section{8,10-diiodo-2,6-dioxo-4 $\lambda^{3}$-ioda-3,5-dioxatricyclo[5.3.1.0 $\left.0^{4,11}\right]$ undeca- 1(11),7,9-triene-9-carboxylic acid}

\section{Crystal data}

$\mathrm{C}_{9} \mathrm{HI}_{3} \mathrm{O}_{6} \cdot 2 \mathrm{H}_{2} \mathrm{O}$

$M_{r}=621.83$

Monoclinic, $C 2 / c$

Hall symbol: $-\mathrm{C} 2 \mathrm{yc}$

$a=14.7667(8) \AA$

$b=11.9890(6) \AA$

$c=9.7419(5) \AA$

$\beta=127.4236(5)^{\circ}$

$V=1369.68(12) \AA^{3}$

$Z=4$

\section{Data collection}

Bruker APEXII CCD area-detector diffractometer

Radiation source: fine-focus sealed tube

Graphite monochromator

$\varphi$ and $\omega$ scans

Absorption correction: multi-scan

(APEX2; Bruker, 2007)

$T_{\min }=0.217, T_{\max }=0.492$
$F(000)=1128$

$D_{\mathrm{x}}=3.016 \mathrm{Mg} \mathrm{m}^{-3}$

Melting point: $573 \mathrm{~K}$

Mo $K \alpha$ radiation, $\lambda=0.71069 \AA$

Cell parameters from 3350 reflections

$\theta=2.4-27.4^{\circ}$

$\mu=6.88 \mathrm{~mm}^{-1}$

$T=130 \mathrm{~K}$

Prism, colourless

$0.32 \times 0.14 \times 0.12 \mathrm{~mm}$

4078 measured reflections

1547 independent reflections

1515 reflections with $I>2 \sigma(I)$

$R_{\text {int }}=0.016$

$\theta_{\text {max }}=27.4^{\circ}, \theta_{\text {min }}=2.4^{\circ}$

$h=-10 \rightarrow 19$

$k=-14 \rightarrow 15$

$l=-12 \rightarrow 12$ 


\section{Refinement}

Refinement on $F^{2}$

Least-squares matrix: full

$R\left[F^{2}>2 \sigma\left(F^{2}\right)\right]=0.042$

$w R\left(F^{2}\right)=0.097$

$S=1.23$

1547 reflections

93 parameters

0 restraints

Primary atom site location: structure-invariant direct methods
Secondary atom site location: difference Fourier map

Hydrogen site location: inferred from neighbouring sites

$\mathrm{H}$-atom parameters constrained

$w=1 /\left[\sigma^{2}\left(F_{\mathrm{o}}^{2}\right)+(0.0157 P)^{2}+40.7765 P\right]$ where $P=\left(F_{\mathrm{o}}{ }^{2}+2 F_{\mathrm{c}}{ }^{2}\right) / 3$

$(\Delta / \sigma)_{\max }<0.001$

$\Delta \rho_{\max }=2.36 \mathrm{e}^{-3}$

$\Delta \rho_{\min }=-2.23$ e $\AA^{-3}$

Special details

Geometry. All esds (except the esd in the dihedral angle between two 1.s. planes) are estimated using the full covariance matrix. The cell esds are taken into account individually in the estimation of esds in distances, angles and torsion angles; correlations between esds in cell parameters are only used when they are defined by crystal symmetry. An approximate (isotropic) treatment of cell esds is used for estimating esds involving l.s. planes.

Refinement. Refinement of $F^{2}$ against ALL reflections. The weighted $R$-factor $w R$ and goodness of fit $S$ are based on $F^{2}$, conventional $R$-factors $R$ are based on $F$, with $F$ set to zero for negative $F^{2}$. The threshold expression of $F^{2}>\sigma\left(F^{2}\right)$ is used only for calculating $R$-factors(gt) etc. and is not relevant to the choice of reflections for refinement. $R$-factors based on $F^{2}$ are statistically about twice as large as those based on $F$, and $R$ - factors based on ALL data will be even larger.

Fractional atomic coordinates and isotropic or equivalent isotropic displacement parameters $\left(\AA^{2}\right)$

\begin{tabular}{llllll}
\hline & $x$ & $y$ & $z$ & $U_{\text {iso }} * U_{\mathrm{eq}}$ & Occ. $(<1)$ \\
\hline $\mathrm{C} 1$ & 0.5000 & $0.3381(8)$ & 0.7500 & $0.0216(19)$ & \\
$\mathrm{C} 2$ & $0.4147(5)$ & $0.3903(6)$ & $0.5979(8)$ & $0.0207(13)$ & \\
$\mathrm{C} 3$ & $0.4155(6)$ & $0.5066(6)$ & $0.5979(9)$ & $0.0249(14)$ & \\
C4 & $0.3340(6)$ & $0.3120(6)$ & $0.4528(9)$ & $0.0248(14)$ & \\
C5 & 0.5000 & $0.5629(8)$ & 0.7500 & $0.0222(19)$ & \\
C6 & 0.5000 & $0.6916(8)$ & 0.7500 & $0.027(2)$ & \\
I1 & 0.5000 & $0.16946(5)$ & 0.7500 & $0.02526(18)$ & \\
I2 & $0.29502(5)$ & $0.59994(5)$ & $0.37929(8)$ & $0.0469(2)$ & \\
O1 & $0.2532(5)$ & $0.3419(5)$ & $0.3093(7)$ & $0.0386(14)$ & \\
O2 & $0.3559(5)$ & $0.2049(5)$ & $0.4946(7)$ & $0.0313(12)$ & \\
O3 & $0.5338(6)$ & $0.7375(5)$ & $0.6751(9)$ & $0.0474(16)$ & \\
H3 & 0.5308 & 0.8054 & 0.6818 & $0.071 *$ & \\
O1W & $0.4370(6)$ & $0.9449(6)$ & $0.5383(9)$ & $0.0494(16)$ & 0.50 \\
H1W & 0.4763 & 0.8910 & 0.5958 & $0.074^{*}$ & 0.50 \\
H2W & 0.4756 & 0.9896 & 0.5303 & $0.074 *$ & \\
H3W & 0.3831 & 0.9240 & 0.4416 & $0.074 *$ & \\
& & & & &
\end{tabular}

Atomic displacement parameters $\left(\AA^{2}\right)$

\begin{tabular}{lllllll}
\hline & $U^{11}$ & $U^{22}$ & $U^{33}$ & $U^{12}$ & $U^{13}$ & $U^{23}$ \\
\hline C1 & $0.021(4)$ & $0.019(4)$ & $0.021(5)$ & 0.000 & $0.011(4)$ & 0.000 \\
C2 & $0.017(3)$ & $0.024(3)$ & $0.012(3)$ & $0.000(2)$ & $0.004(2)$ & $-0.001(2)$ \\
C3 & $0.021(3)$ & $0.030(4)$ & $0.017(3)$ & $0.005(3)$ & $0.008(3)$ & $0.004(3)$ \\
C4 & $0.021(3)$ & $0.031(4)$ & $0.015(3)$ & $-0.002(3)$ & $0.007(3)$ & $-0.003(3)$ \\
C5 & $0.026(5)$ & $0.018(4)$ & $0.029(5)$ & 0.000 & $0.020(4)$ & 0.000
\end{tabular}


supporting information

\begin{tabular}{lllllll} 
C6 & $0.039(6)$ & $0.012(4)$ & $0.035(6)$ & 0.000 & $0.025(5)$ & 0.000 \\
I1 & $0.0282(3)$ & $0.0169(3)$ & $0.0269(3)$ & 0.000 & $0.0148(3)$ & 0.000 \\
I2 & $0.0427(4)$ & $0.0392(3)$ & $0.0341(3)$ & $0.0125(2)$ & $0.0104(3)$ & $0.0160(2)$ \\
O1 & $0.031(3)$ & $0.042(3)$ & $0.019(3)$ & $0.000(3)$ & $0.003(2)$ & $-0.004(2)$ \\
O2 & $0.030(3)$ & $0.029(3)$ & $0.024(3)$ & $-0.008(2)$ & $0.011(2)$ & $-0.008(2)$ \\
O3 & $0.074(5)$ & $0.028(3)$ & $0.069(5)$ & $0.000(3)$ & $0.058(4)$ & $0.005(3)$ \\
O1W & $0.050(4)$ & $0.044(4)$ & $0.048(4)$ & $-0.008(3)$ & $0.026(3)$ & $-0.002(3)$ \\
\hline
\end{tabular}

Geometric parameters $\left(\AA,{ }^{\circ}\right)$

\begin{tabular}{|c|c|c|c|}
\hline $\mathrm{C} 1-\mathrm{C} 2^{\mathrm{i}}$ & $1.380(8)$ & $\mathrm{C} 5-\mathrm{C} 3^{\mathrm{i}}$ & $1.400(8)$ \\
\hline $\mathrm{C} 1-\mathrm{I} 1$ & $2.022(9)$ & $\mathrm{C} 5-\mathrm{C} 6$ & $1.543(13)$ \\
\hline $\mathrm{C} 2-\mathrm{C} 3$ & $1.394(10)$ & $\mathrm{C} 6-\mathrm{O} 3$ & $1.237(7)$ \\
\hline $\mathrm{C} 2-\mathrm{C} 4$ & $1.501(9)$ & $\mathrm{I} 1-\mathrm{O} 2$ & $2.113(5)$ \\
\hline $\mathrm{C} 3-\mathrm{C} 5$ & $1.400(8)$ & $\mathrm{O} 3-\mathrm{H} 3$ & 0.8200 \\
\hline $\mathrm{C} 3-\mathrm{I} 2$ & $2.085(7)$ & $\mathrm{O} 1 \mathrm{~W}-\mathrm{H} 1 \mathrm{~W}$ & 0.8201 \\
\hline $\mathrm{C} 4-\mathrm{O} 1$ & $1.216(9)$ & $\mathrm{O} 1 \mathrm{~W}-\mathrm{H} 2 \mathrm{~W}$ & 0.8200 \\
\hline $\mathrm{C} 4-\mathrm{O} 2$ & $1.326(9)$ & $\mathrm{O} 1 \mathrm{~W}-\mathrm{H} 3 \mathrm{~W}$ & 0.8201 \\
\hline $\mathrm{C} 4 \cdots \mathrm{O} 3^{\mathrm{ii}}$ & $2.944(10)$ & $\mathrm{I} 2 \cdots \mathrm{O} 2^{\mathrm{iv}}$ & $3.156(5)$ \\
\hline $\mathrm{I} 1 \cdots \mathrm{O} 1 \mathrm{~W}^{\mathrm{iii}}$ & $3.173(7)$ & $\mathrm{I} 2 \cdots \mathrm{O} 1^{\text {iv }}$ & $3.274(6)$ \\
\hline $\mathrm{C} 2 \mathrm{i}-\mathrm{C} 1-\mathrm{C} 2$ & $126.1(9)$ & $\mathrm{C} 3-\mathrm{C} 5-\mathrm{C} 3^{\mathrm{i}}$ & $122.3(9)$ \\
\hline $\mathrm{C} 2 \mathrm{i}-\mathrm{C} 1-\mathrm{I} 1$ & $117.0(5)$ & $\mathrm{C} 3-\mathrm{C} 5-\mathrm{C} 6$ & $118.8(5)$ \\
\hline $\mathrm{C} 2-\mathrm{C} 1-\mathrm{I} 1$ & $117.0(5)$ & $\mathrm{O} 33^{\mathrm{i}}-\mathrm{C} 6-\mathrm{O} 3$ & $127.1(10)$ \\
\hline $\mathrm{C} 1-\mathrm{C} 2-\mathrm{C} 3$ & $116.8(6)$ & $\mathrm{O} 3-\mathrm{C} 6-\mathrm{C} 5$ & $116.5(5)$ \\
\hline $\mathrm{C} 1-\mathrm{C} 2-\mathrm{C} 4$ & $114.3(6)$ & $\mathrm{C} 1-\mathrm{I} 1-\mathrm{O} 2$ & $78.38(15)$ \\
\hline $\mathrm{C} 3-\mathrm{C} 2-\mathrm{C} 4$ & $128.9(6)$ & $\mathrm{O} 2 \mathrm{i}-\mathrm{I} 1-\mathrm{O} 2$ & $156.8(3)$ \\
\hline $\mathrm{C} 2-\mathrm{C} 3-\mathrm{C} 5$ & $119.0(7)$ & $\mathrm{C} 4-\mathrm{O} 2-\mathrm{I} 1$ & $116.1(4)$ \\
\hline $\mathrm{C} 2-\mathrm{C} 3-\mathrm{I} 2$ & $122.3(5)$ & $\mathrm{C} 6-\mathrm{O} 3-\mathrm{H} 3$ & 109.5 \\
\hline $\mathrm{C} 5-\mathrm{C} 3-\mathrm{I} 2$ & $118.7(6)$ & $\mathrm{H} 1 \mathrm{~W}-\mathrm{O} 1 \mathrm{~W}-\mathrm{H} 2 \mathrm{~W}$ & 109.6 \\
\hline $\mathrm{O} 1-\mathrm{C} 4-\mathrm{O} 2$ & $121.6(7)$ & $\mathrm{H} 1 \mathrm{~W}-\mathrm{O} 1 \mathrm{~W}-\mathrm{H} 3 \mathrm{~W}$ & 109.5 \\
\hline $\mathrm{O} 1-\mathrm{C} 4-\mathrm{C} 2$ & $124.1(7)$ & $\mathrm{H} 2 \mathrm{~W}-\mathrm{O} 1 \mathrm{~W}-\mathrm{H} 3 \mathrm{~W}$ & 109.6 \\
\hline $\mathrm{O} 2-\mathrm{C} 4-\mathrm{C} 2$ & $114.2(6)$ & & \\
\hline $\mathrm{C} 2-\mathrm{C} 1-\mathrm{C} 2-\mathrm{C} 3$ & $0.5(5)$ & $\mathrm{I} 2-\mathrm{C} 3-\mathrm{C} 5-\mathrm{C} 3^{\mathrm{i}}$ & $-179.6(5)$ \\
\hline $\mathrm{I} 1-\mathrm{C} 1-\mathrm{C} 2-\mathrm{C} 3$ & $-179.5(5)$ & $\mathrm{C} 2-\mathrm{C} 3-\mathrm{C} 5-\mathrm{C} 6$ & $-179.5(5)$ \\
\hline $\mathrm{C} 2-\mathrm{C} 1-\mathrm{C} 2-\mathrm{C} 4$ & $179.7(6)$ & $\mathrm{I} 2-\mathrm{C} 3-\mathrm{C} 5-\mathrm{C} 6$ & $0.4(5)$ \\
\hline $\mathrm{I} 1-\mathrm{C} 1-\mathrm{C} 2-\mathrm{C} 4$ & $-0.3(6)$ & $\mathrm{C} 3-\mathrm{C} 5-\mathrm{C} 6-\mathrm{O}^{\mathrm{i}}$ & $105.1(5)$ \\
\hline $\mathrm{C} 1-\mathrm{C} 2-\mathrm{C} 3-\mathrm{C} 5$ & $-0.9(9)$ & $\mathrm{C} 3-\mathrm{C} 5-\mathrm{C} 6-\mathrm{O} 3$ & $-74.9(5)$ \\
\hline $\mathrm{C} 4-\mathrm{C} 2-\mathrm{C} 3-\mathrm{C} 5$ & $180.0(6)$ & $\mathrm{C} 3 \mathrm{i}-\mathrm{C} 5-\mathrm{C} 6-\mathrm{O} 3$ & $105.1(5)$ \\
\hline $\mathrm{C} 1-\mathrm{C} 2-\mathrm{C} 3-\mathrm{I} 2$ & $179.1(4)$ & $\mathrm{C} 2 \mathrm{i}-\mathrm{C} 1-\mathrm{I} 1-\mathrm{O} 2$ & $179.6(4)$ \\
\hline $\mathrm{C} 4-\mathrm{C} 2-\mathrm{C} 3-\mathrm{I} 2$ & $0.1(11)$ & $\mathrm{C} 2-\mathrm{C} 1-\mathrm{I} 1-\mathrm{O} 2$ & $-0.4(4)$ \\
\hline $\mathrm{C} 1-\mathrm{C} 2-\mathrm{C} 4-\mathrm{O} 1$ & $179.7(7)$ & $\mathrm{O} 1-\mathrm{C} 4-\mathrm{O} 2-\mathrm{I} 1$ & $180.0(6)$ \\
\hline $\mathrm{C} 3-\mathrm{C} 2-\mathrm{C} 4-\mathrm{O} 1$ & $-1.3(13)$ & $\mathrm{C} 2-\mathrm{C} 4-\mathrm{O} 2-\mathrm{I} 1$ & $-1.5(8)$ \\
\hline $\mathrm{C} 1-\mathrm{C} 2-\mathrm{C} 4-\mathrm{O} 2$ & $1.2(9)$ & $\mathrm{C} 1-\mathrm{I} 1-\mathrm{O} 2-\mathrm{C} 4$ & $1.1(5)$ \\
\hline
\end{tabular}




\section{supporting information}

$\mathrm{C} 3-\mathrm{C} 2-\mathrm{C} 4-\mathrm{O} 2 \quad-179.7(7) \quad \mathrm{O} 2-\mathrm{I} 1-\mathrm{O} 2-\mathrm{C} 4$

$\mathrm{C} 2-\mathrm{C} 3-\mathrm{C} 5-\mathrm{C}^{\mathrm{i}} \quad 0.5(5)$

Symmetry codes: (i) $-x+1, y,-z+3 / 2$; (ii) $-x+1,-y+1,-z+1$; (iii) $x, y-1, z$; (iv) $-x+1 / 2, y+1 / 2,-z+1 / 2$.

Hydrogen-bond geometry $\left(\AA,{ }^{\circ}\right)$

\begin{tabular}{lllll}
\hline$D-\mathrm{H} \cdots A$ & $D-\mathrm{H}$ & $\mathrm{H} \cdots A$ & $D \cdots A$ & $D-\mathrm{H} \cdots A$ \\
\hline $\mathrm{O} 3-\mathrm{H} 3 \cdots \mathrm{O} 1 W$ & 0.82 & 2.08 & $2.772(9)$ & 142 \\
$\mathrm{O} 1 W-\mathrm{H} 1 W \cdots \mathrm{O} 3$ & 0.82 & 1.98 & $2.772(9)$ & 163 \\
$\mathrm{O} 1 W-\mathrm{H} 2 W \cdots \mathrm{O} 1 W^{v}$ & 0.82 & 1.94 & $2.730(14)$ & 160 \\
$\mathrm{O} 1 W-\mathrm{H} 3 W \cdots \mathrm{O} 1^{\text {iv }}$ & 0.82 & 2.24 & $3.053(9)$ & 172 \\
\hline
\end{tabular}

Symmetry codes: (iv) $-x+1 / 2, y+1 / 2,-z+1 / 2 ;$ (v) $-x+1,-y+2,-z+1$. 